\title{
Modeling of Location Estimation for Object Tracking in WSN
}

\author{
Hung-Chi Chu, ${ }^{1}$ Tsung-Han Lee, ${ }^{2}$ Lin-huang Chang, ${ }^{2}$ and Chung-Jie Li ${ }^{1}$ \\ ${ }^{1}$ Department of Information and Communication Engineering, Chaoyang University of Technology, No. 168, \\ Ji-Fong East Road, Wu-Fong District, Taichung 41349, Taiwan \\ ${ }^{2}$ Department of Computer Science, National Taichung University of Education, No. 140, Min-Sheng Road, Taichung 403, Taiwan
}

Correspondence should be addressed to Lin-huang Chang; albertchang04@gmail.com

Received 21 June 2013; Accepted 16 August 2013

Academic Editor: Chih-Hao Lin

Copyright (c) 2013 Hung-Chi Chu et al. This is an open access article distributed under the Creative Commons Attribution License, which permits unrestricted use, distribution, and reproduction in any medium, provided the original work is properly cited.

Location estimation for object tracking is one of the important topics in the research of wireless sensor networks (WSNs). Recently, many location estimation or position schemes in WSN have been proposed. In this paper, we will propose the procedure and modeling of location estimation for object tracking in WSN. The designed modeling is a simple scheme without complex processing. We will use Matlab to conduct the simulation and numerical analyses to find the optimal modeling variables. The analyses with different variables will include object moving model, sensing radius, model weighting value $\alpha$, and power-level increasing ratio $k$ of neighboring sensor nodes. For practical consideration, we will also carry out the shadowing model for analysis.

\section{Introduction}

Recently, with the characteristics of low cost and low power consumption, wireless sensor net-work (WSN) communication has attracted lots of attentions on different applications and services, such as monitoring and object tracking. Location estimation for object tracking is one of the important topics in the research of wireless sensor networks (WSNs) $[1,2]$. The wireless sensors not only are able to induce and detect the environmental target and the change of environment but also deal with collected data and send the disposed information to the sink or base station via wireless communication.

For WSN applications, tracking moving objects is one of the important issues. Tracking moving objects is more difficult than sensing objects in fixed area because the objects may move from time to time, and sufficient computing power and storage space are required for disposing information of objects and sending the information to users. In the application of wireless sensor networks, users hope to use sensors to collect needed information, such as temperature, gas concentration, position of wildlife, and so forth. For users, the position of object is an important piece of information. For example, in an intelligent building, when sensors detect a fire event, the firemen wish to know the fire site and moving direction through the sensor nodes so that the action can be carried out promptly. Therefore, to acquire the accurate position of the object is one of issues concerning object tracking.

Another important research issue is information processing. Since the object may move everywhere at any moment, the information of object should be updated and sent to the sink timely so that we can maintain the object location accurately. If the monitoring area is very large, the sensing coverage and data aggregation technology needs to be taken into consideration to save energy in terms of sensing and transmission. Therefore, in the paper we will propose the procedure and modeling of location estimation for object tracking with energy issues in mind while maintaining the relatively low estimation error.

The rest of this paper is organized as follows. Section 2 describes the research related to the positioning and location estimation for object tracking. The detailed design and modeling of the proposed scheme is illustrated in Section 3. Section 4 provides the analyses and evaluations as well as discussions based on the simulation and numerical results. We summarize this paper and address future work in Section 5. 


\section{Related Works}

Location estimation of object tracking is to define the object movement path and position [3-7]. In recent years, global positioning system (GPS) [8], implemented in real system, is one of the most popular positioning systems for outdoor environment. The average error of GPS is within $3 \mathrm{~m}$. However, the GPS positioning technology is not suitable for wireless sensor networks due to size, cost, and power consumption constraints.

The location estimation for radio communication can be divided into 2 categories: range-based positioning [911] and range-free positioning [12, 13]. The range-based positioning technologies include angle of arrival (AOA) [9], time of arrival (TOA) [9], time difference of arrival (TDOA) [9], and received signal strength indicator (RSSI) [11]. The range-based technology in general can obtain more accurate location estimation by applying the distance and angle information received from specific or expensive network equipments; however, it might not be suitable for large-scale deployment of WSNs.

The range-free positioning technology, on the other hand, estimates the location without the assist from any expensive network equipment. The centroid positioning mechanism [13] employed the centroid of selected reference points to estimate its own position. The general scheme for object tracking is taking the object as the center of a circle and estimating the signal radius with the consideration of moving speed. Besides this general scheme, some other researches $[14,15]$ have been studied. The research in [14] proposed a method using a mobile agent to track the object moving path and three fixed sensor nodes to calculate the object position. These three sensor nodes broadcast a message to their neighboring nodes for object detection. In order to obtain more accurate object position, some researches $[3,4]$ defined some sensor nodes within certain range from the object to help tracking the object. Other sensor nodes beyond this range maintain in dormant states. The research in [15] proposed the dynamic adjustment of the signal coverage based on the object behavior to reduce the tracking area in wireless sensor networks. The research can track the object with minimum number of sensor nodes so that the network lifetime can be prolonged.

The deployment of WSNs usually consists of a great number of sensors. To manage the sensor node resources and collected data, some schemes need to be taken into account to provide efficient data processing or message transfer, especially in large-scale WSNs. In general, there are two types of information processing in WSNs, named distributed processing $[12,13,16,17]$ and centralized processing $[14,18-$ 21]. For the distributed processing, when the sensor nodes sense and collect data, they will calculate or process the data followed by sending the data to the sink. GPS system is one typical position system using distributed processing [8]. GPS estimates more accurate location; however, it takes a longer time to first fix (TTFF) and incurs the additional cost of setting up a GPS receiver for each sensor node. Some other researches using related positions of the nodes [16] or areabased position schemes [13] for distributed data processing were also proposed. The research in [12] narrows down the possible region, formed by selecting three anchors among all sensing nodes, in which a particular node may reside. The location of an object can be determined by the center of gravity of the intersection of triangles. To reduce the number of anchors, the research in [17] employed a few mobile anchors equipped with the GPS capability to broadcast their current positions periodically for location estimation.

On the other hand in centralized processing, upon receiving the sensed data, the sensor nodes send it to the sink via some routing protocols for data processing. The data aggregation technology [18-21] might be employed before the data reach the sink so as to reduce the amount of data transfer and consequently provide the energy saving. The research in [14] set a data volume threshold for the detected object data in WSNs. When the data volume detected by an agent node is smaller than the threshold, the agent node will send the data to the sink directly. If the collected data volume is larger than the threshold, the agent node will carry out the data fusion for all collected data and then send them to the sink.

From the related researches discussed above, not all sensor nodes can afford the GPS capability due to the limitations of sensor nodes in size, cost, and power consumption. Also due to the limited computational power of sensor nodes, simpler positioning and data processing mechanisms will be the major consideration in this paper. Therefore, this paper aims at the scenario wherein static sensing nodes are deployed in fixed location. The proposed modeling of location estimation for object tracking developed using rangefree positioning technology based on centroid scheme [13] as well as centralized data processing technology to reduce the data processing and traffic loads is suitable for largescale WSNs. We will also use data aggregation idea to reduce the data volume transfer between the sensing nodes and sink. From the result of related researches above, the rangefree positioning technology combined with data aggregation used in the proposed mechanism is suitable for practical use in large-scale WSNs which are constrained in terms of energy consumption, computation power, and device cost. Furthermore, the proposed mechanism using dynamic sensing procedure with different sensing radii and powerlevel of the transmission signal will improve the positioning accuracy as compared to other related schemes.

\section{Proposed Location Estimation Scheme}

3.1. Dynamic Sensing Procedure. In this paper, we will make the following assumptions for our location estimation model. First, we assume that the WSN nodes deployment is in a 2dimensional plane. Secondly, there is no interference between any two sensor nodes. Thirdly, all sensor nodes in WSN have the knowledge of their own IDs and corresponding GPS positions as well as sink GPS value. Lastly, we assume that every node knows its neighboring nodes' ID. All the above mentioned data can be set up or acquired during the initial deployment of the WSN. The sink node maintains the record of IDs and GPS positions of all sensor nodes. Within the sensing range, $\mathrm{R}$, each sensor node can detect the movement 


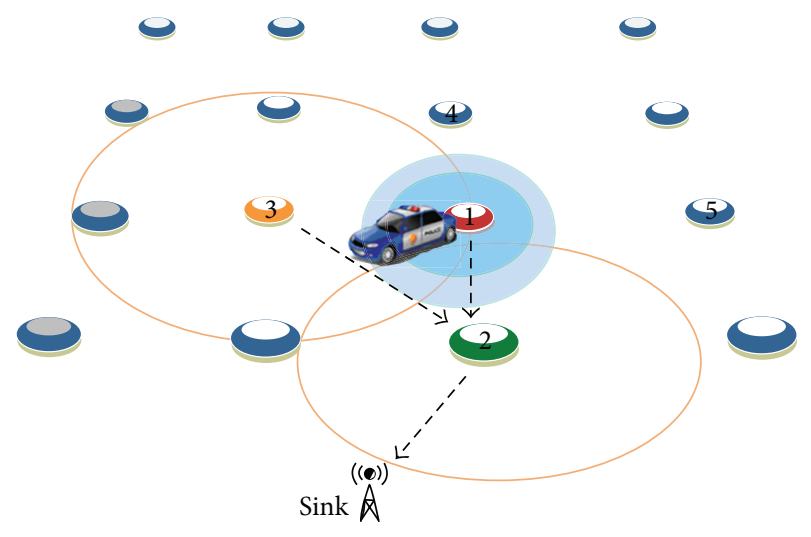

FIgURE 1: The dynamic power-level sensing topology.

of the object. In this research, we deploy a square network nodes topology to provide fully coverage of sensing network. The proposed location estimation for object tracking in WSN includes three steps.

Step 1. Dynamic power-level sensing: once detecting the appearance of one object, the sensor node informs the onehop neighboring node to increase the power level for sensing.

Step 2. Cluster head selection: designate a node from the sensing nodes which is the closest node to the sink. The selected node, named cluster head, will collect all data from sensing nodes and then send the data to the sink.

Step 3. Modeling of the location estimation: upon receiving the data from the cluster head, the sink calculates the object location using the location estimation model.

The detailed modeling and mechanism of the proposed location estimation for object tracking is described below.

While estimating the position of moving object in WSN, the sensing node calculates the position according to models such as [13]. In general, using more sensing nodes to detect the object, the estimated location will be more accurate. To increase the accuracy of location estimation for object tracking, the first step in our proposed mechanism is to dynamically adjust the sensing power-level of neighboring nodes which is one hop away from the initial sensing node. The one-hop neighboring nodes increase their power by $k$ time to extend their corresponding signal coverage. The dynamic power-level sensing topology and scheme is illustrated in Figure 1, where sensor node 1 is the initial sensing node to detect an object. Once detecting the appearance of the object, the sensing node, node 1 , issues a message, with TTL setting as 1 , to its one-hop neighboring nodes, nodes 2 , 3,4 , and 5 as shown in Figure 1. Upon receiving the message from the initial sensing node, the one-hop neighboring nodes extend their corresponding signal coverage by increasing the sensing power-level to $k$ time. The moving object location can be calculated using location estimation model, described later, from all sensible nodes corresponding to nodes 1, 2, and 3 as shown in Figure 1 for example.
The next step is to send sensed information back to the sink node by each sensible node. In this research, we designate a node from the sensible nodes being the closest node to the sink as the cluster head. The cluster head will collect all data from sensible nodes and then send the data to the sink. This will reduce the unnecessary messages sending from other sensible nodes, except the cluster head, to the sink.

Once the cluster head is determined, all other sensible nodes will send the sensing information to the cluster head. After the data fusing, the cluster head sends the sensing object information to the sink. Upon receiving the data from the cluster head, the sink calculates the object location using the location estimation model. The modeling of location estimation for object tracking is described in more details in Section 3.2.

3.2. Modeling of Location Estimation for Object Tracking. Because the dynamic power-level sensing is applied to the neighboring sensor nodes which would be different from the initial sensor node, we define the initial sensor node as major node, node 1 in Figure 1, and the other sensible neighboring nodes as minor nodes, for example, nodes 2 and 3 in Figure 1. The algorithm with distance formula for our location estimation is illustrated in Algorithm 1.

In our proposed algorithm, there are two types of sensing data between sensor nodes to the cluster head. For those nodes without changing the sensing range, they will send an Mposition (cluster, ID) packet to the cluster head, where cluster is the cluster head sensor ID, and ID is the sensor node ID. If a sensor node, increasing its power by $k$ time, detects the object, it will send an Nposition (cluster, ID) packet to the cluster head. After receiving the information from all sensible nodes, the cluster head processes and compresses the data and then sends it to the sink. Upon receiving the data from the cluster, the sink calculates the object location according to the following equation:

$$
\begin{aligned}
& \left(O_{x}, O_{y}\right) \\
& \quad=\left(\frac{\alpha \sum_{i=1}^{z} M_{x_{i}}+\sum_{i=1}^{j} N_{x_{i}}}{\alpha z+j}, \frac{\alpha \sum_{i=1}^{z} M_{y_{i}}+\sum_{i=1}^{j} N_{y_{i}}}{\alpha z+j}\right),
\end{aligned}
$$

where the $\left(O_{x}, O_{y}\right),\left(M_{x}, M_{y}\right)$, and $\left(N_{x}, N_{y}\right)$ are the coordinate of object, major node, and minor node, respectively. Also, the $z$ and $j$ are the numbers of the major nodes and minor nodes, respectively. In (1), we add a weighting value $\alpha$ to the estimated formula because sensing ranges for major nodes and minor nodes are different. The $\alpha$ value could be related to the increase of the power level $k$ for minor nodes. When we conduct the performance analysis, we compare the numerical result for different $k$ and $\alpha$ values. The algorithm for our proposed location estimation model is shown in Algorithm 2.

Since the practical wireless communications will suffer from all kinds of interference and signal fading issue, the idea model with circular sensing coverage may not be feasible. Therefore, we further consider the shadowing model in our 
(1) // Given a graph $G=(V, E)$

(2) $/ / V$ presents the set of sensor nodes

(3) // E presents the set of communication link between sensor node and its neighbors

(4) // MPosition $\left(M_{x}, M_{y}\right)$ is the coordinator of the major node

(5) // NPosition $\left(N_{x}, N_{y}\right)$ is the coordinator of the minor node

(6) // $R$ presents the sensing range of sensor node

(7) $S=\left\{s_{a}\right\}$, for $a=1,2, \ldots, i$, and $S \in V \quad / / S$ presents the set of sensor nodes detecting object $O$

(8) $N=\left\{n_{b}\right\}$, for $b=1,2, \ldots, j$, and $N \in V \quad / / N$ presents the set of node's neighboring nodes with enlarged power,

(9) $\operatorname{dist}\left(O, s_{a}\right)=\sqrt{\left(O_{x}-s_{a_{x}}\right)^{2}+\left(O_{y}-s_{a_{y}}\right)^{2}}$ which can detect object $O$

(10) if $\operatorname{dist}\left(O, s_{a}\right)<R$ then // The proposed method will be triggered when a sensor node $S_{a}$ detects an object $O$.

(11) $\operatorname{dist}\left(O, n_{b}\right)=\sqrt{\left(O_{x}-n_{b_{x}}\right)^{2}+\left(O_{y}-n_{b_{y}}\right)^{2}} / /$ In order to determine object's location, the sensor node notifies

(12) end if its one-hop neighboring nodes to increase their sensing range.

Algorithm 1: Algorithm with distance formula for the proposed location estimation.

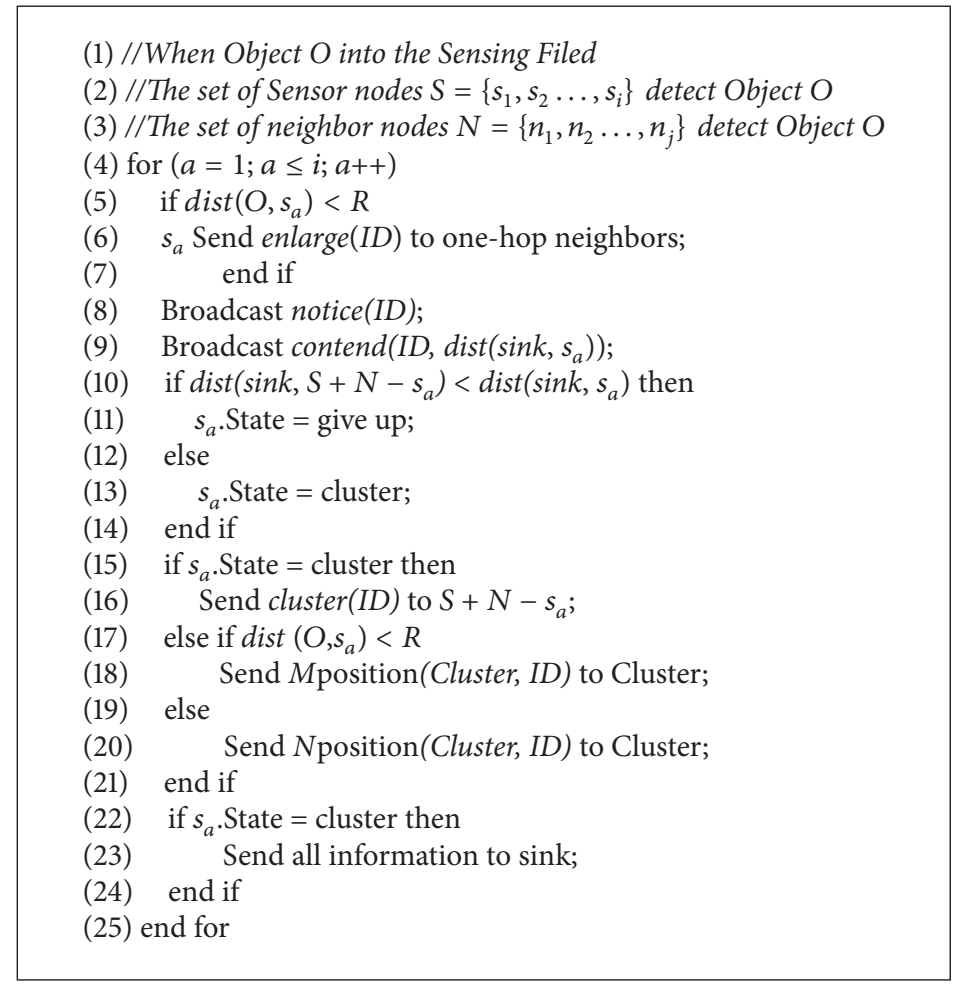

Algorithm 2: Algorithm of the proposed location estimation model.

design and analysis. We model the shadowing issue with random process. Basically, when the object is close to the sensor node, the sensed probability would be higher than that of the object being remote. The shadowing model applied in our study is shown in the following:

$$
\left[\frac{P_{r}(d)}{P_{r}\left(d_{0}\right)}\right]_{d b}=-10 \beta \log \left(\frac{d}{d_{0}}\right)+X_{d B},
$$

where $X_{d b}$ is the random variable with Gaussian distribution, $\beta$ is the path loss exponent, $d$ is the distance between the object and the sensing node, and $d_{0}$ is the sensing radius.

\section{Numerical Analyses}

The performance analysis is conducted by using Matlab software and the experimental parameters; setup and mobility 


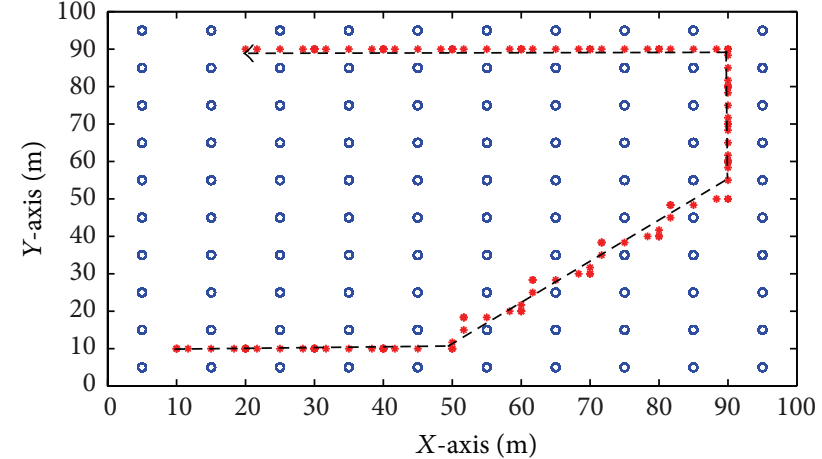

FIGURE 2: Tracking of moving object with line movement path 1 (black broken lines are the real movements, and red dots stand for predicted object locations).

models are defined according to the survey in [22]. We deployed 100 sensor nodes in a $100 \mathrm{~m} \times 100 \mathrm{~m}$ environment. The sensor nodes are equally distributed with square shape in the experimental region. The distance between two neighboring nodes is 10 meters. Two object moving paths are considered in the experiment. Path 1 is line movement with $1 \mathrm{~m} / \mathrm{s}$ moving speed and path 2 corresponds to the random movement with $1-5 \mathrm{~m} / \mathrm{s}$ moving speed. The initial sensing radius $R$ is set as $8 \mathrm{~m}$, which will be varied for different simulations. The experiment time for the simulation is $180 \mathrm{sec}$.

The experiments include object moving model, sensing radius, modeling weight value $\alpha$ and power level increasing ratio $k$ of neighboring sensor nodes. The detailed result for each experiment is discussed in Section 4.1.

4.1. Different Object Moving Models. Figures 2 and 3 illustrate the tracking of moving object with line movement path 1 and random movement path 2, respectively. The location estimation from our model for both path 1 and path 2 are quite close to the real object moving position. The estimations error for path 1 movement is shown in Figure 4 where the $x$-axis represents the simulation time which corresponds to the object position after movement at each instance. The $y$ axis in Figure 4 on the other hand stands for the estimation error between the real object position and calculated position. The average estimated error for path 1 movement is $1.17 \mathrm{~m}$ with $2.83 \mathrm{~m}$ maximum error and standard deviation 0.83 . The occurrence of maximum error appears during 35-80 sec simulation time which proceeds with oblique movement instead of horizontal or vertical movements. For rectangular deployment of sensor nodes, the oblique movement of object is expected to come out with large estimation error.

On the other hand, the estimation error for path 2 movement is illustrated in Figure 5. The average estimated error for path 2 random movement is $1.34 \mathrm{~m}$ with $3.48 \mathrm{~m}$ maximum error and standard deviation 0.62 . The random direction characteristics and various moving speeds is the major reason which causes larger estimated error.

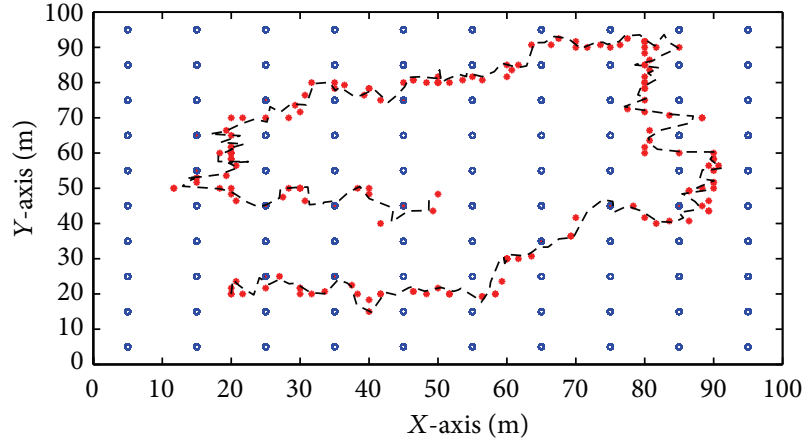

FIGURE 3: Tracking of moving object with random movement path 2 (black broken lines are the real movements, and red dots stand for predicted object locations).

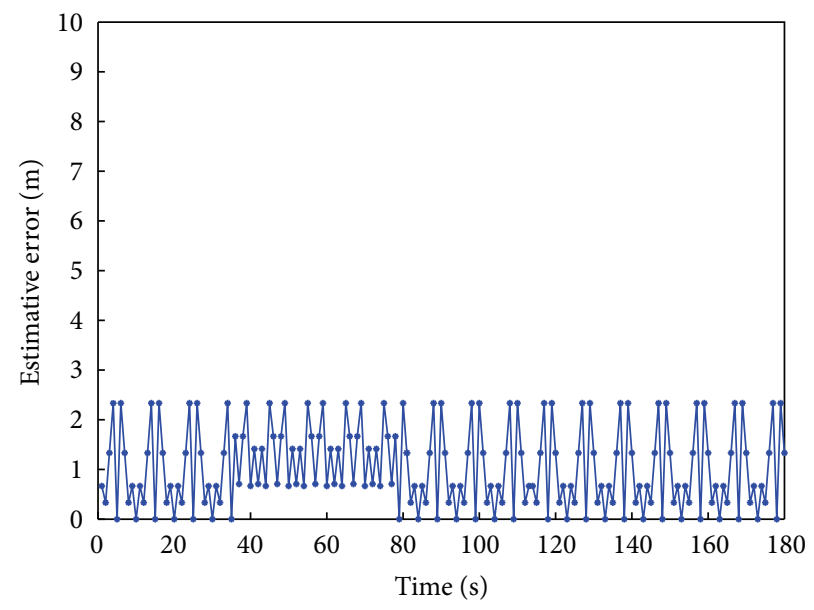

FIgURE 4: The estimation error for path 1 movement.

4.2. Different Sensing Radii. In general, the difference in sensing radius, resulting in different sensing coverage and consequently different signal overlay area, will affect the accuracy of the location estimation. The change of sensing radius will also affect the numbers of major and minor sensor nodes as well as the estimated position. Figures 6 and 7 show the average estimated error with different sensing radii for path 1 and path 2 movement, respectively. In this research with $10 \mathrm{~m}$ separation between each neighboring sensor node, the minimum sensing range would be $7.2 \mathrm{~m}$ in order to fully cover the whole experimental environment. However, for the practical deployment while considering the shadowing effects, it is better to provide sensing radius larger than $8 \mathrm{~m}$. On the other hand, larger sensing radius with larger sensing power will introduce the power consumption issue for WSN applications. From the power energy viewpoint, the sensing radius in this design could be between $8 \mathrm{~m}$ to $10 \mathrm{~m}$.

As shown from the simulation results in Figure 6, when the sensing radius is between $11.2-11.3 \mathrm{~m}$, the location estimation error reaches the minimum. However, by taking account the energy consumption issue, the sensing radius around $9.7 \mathrm{~m}$ with relatively low location estimation error, not larger than $0.1 \mathrm{~m}$ as compared to the sensing radius $11.4 \mathrm{~m}$ with 


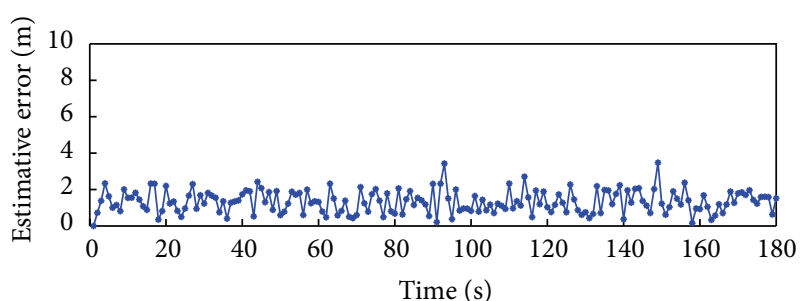

FIGURE 5: The estimation error for path 2 random movement.

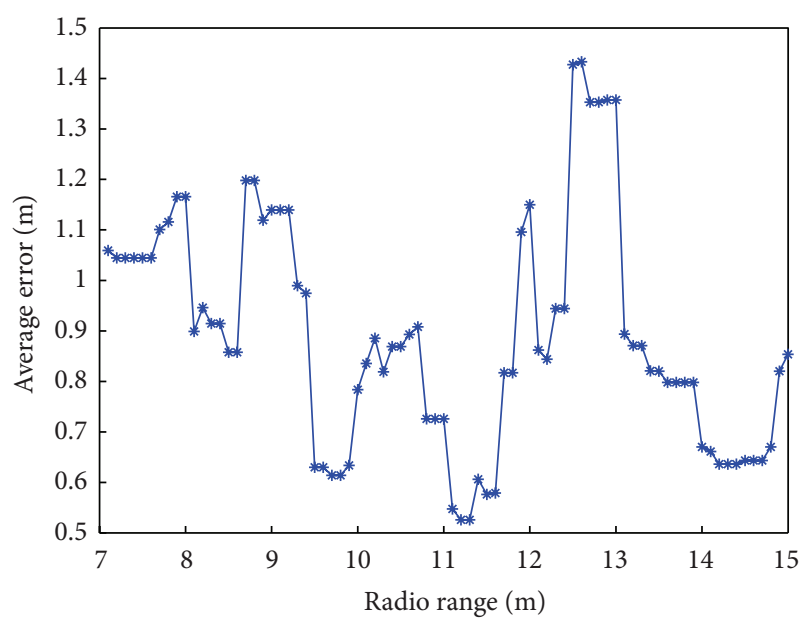

FIGURE 6: The average estimated error with different sensing radii for path 1 movement.

minimum estimation error, could be the optimal candidate in the experimental scenario for path 1 simulation. On the other hand, when the sensing radius is around $11.4 \mathrm{~m}$, the location estimation error reaches the minimum for path 2 case shown in Figure 7. Similarly, we will set the sensing radius $9.6 \mathrm{~m}$ as the optimal candidate for energy consumption consideration in path 2 simulation.

With the optimal sensing radius in mind, more accuracy location estimation could be obtained. Figures 8 and 9 show the estimation error result with optimal sensing radius for path 1 and path 2 movement, respectively. As shown in Figure 8 for path 1 case, the average estimation error is about $0.61 \mathrm{~m}$ with 1.04 maximum error and standard deviation 0.46 which are much smaller than the results with $8 \mathrm{~m}$ sensing radius. Similarly, for the Path 2 movement case in Figure 9 we obtain the average estimation error about $1.03 \mathrm{~m}$ with $3.29 \mathrm{~m}$ maximum error and standard deviation 0.66 . The average estimation error with optimal sensing radius is less than the result with $8 \mathrm{~m}$ sensing radius.

4.3. Different Modeling Weight Values. The weight value $\alpha$ of major sensor node will be analyzed in this subsection. Figures 10 and 11 show the dependence of weighting value on the average location estimation error for path 1 movement with sensing radius $9.7 \mathrm{~m}$ and path 2 movement with sensing radius $9.6 \mathrm{~m}$, respectively. As shown in Figure 10 for path 1 case, for weighting value between 2 and 3 we will obtain the

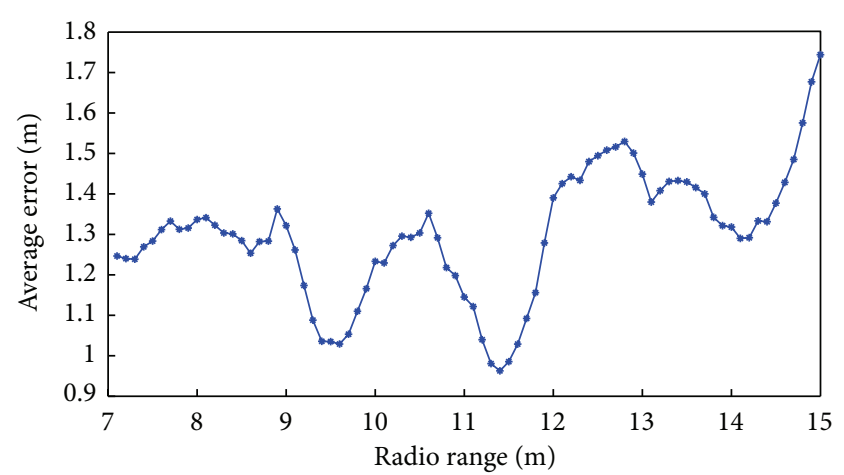

FIGURE 7: The average estimated error with different sensing radii for path 2 movement.

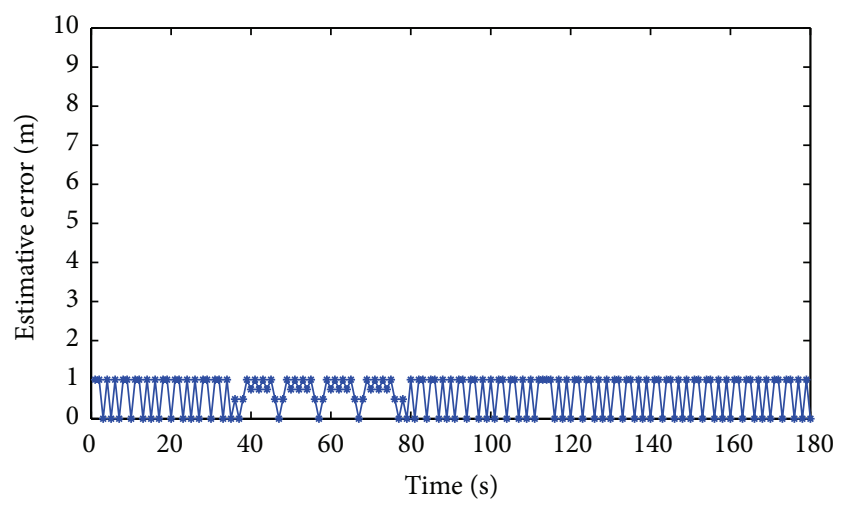

FIGURE 8: The estimation error result with optimal sensing radius for path 1 movement.

lowest estimation error. Similarly, we will obtain the relatively low estimation error for weighting value between 2 and 3 in Figure 11 path 2 case. In general, large weighting value (larger than 3) would push the calculation of location estimation too close to major sensor node. On the other hand, small weighting value (smaller than 2 ) would push the calculation of location estimation too close to minor sensor node. Both situations would increase the estimation error.

4.4. Different Power-Level Increasing Ratios. Figure 12 shows the simulation results of average estimation error with different sensing radii and power-level increasing ratios $k$ for path 1 movement. As shown in Figure 12, when $k$ value of power-level increasing ratio for minor sensor node is larger than 2, the estimation error becomes relatively large. This is because the remote sensor nodes with extended sensing coverage may detect the object and consequently result in the increase of the estimation error. The detailed estimation errors with corresponding variables for some instances of path 1 movement are listed in Table 1. Although the minimum estimation error is obtained with $9.9-10 \mathrm{~m}$ sensing radius and $k=1.7$, the optimal selection would be the case with $9.7 \mathrm{~m}$ sensing radius and $k=1.5$ by considering the energy consumption issue. 


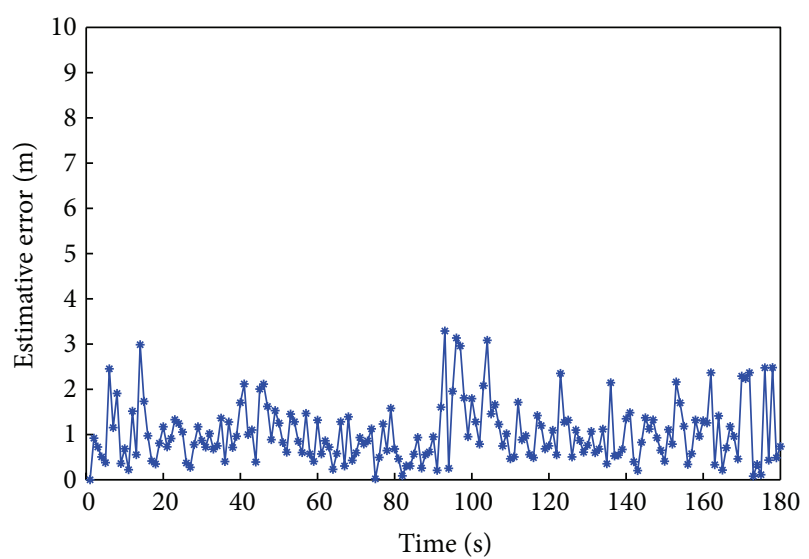

FIGURE 9: The estimation error result with optimal sensing radius for path 2 movement.

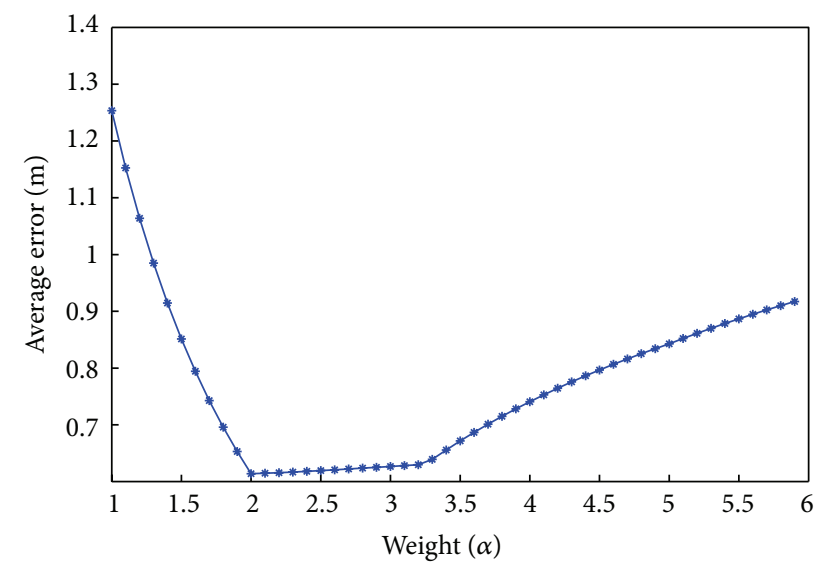

Figure 10: The dependence of weighting value on the average location estimation error for path 1.

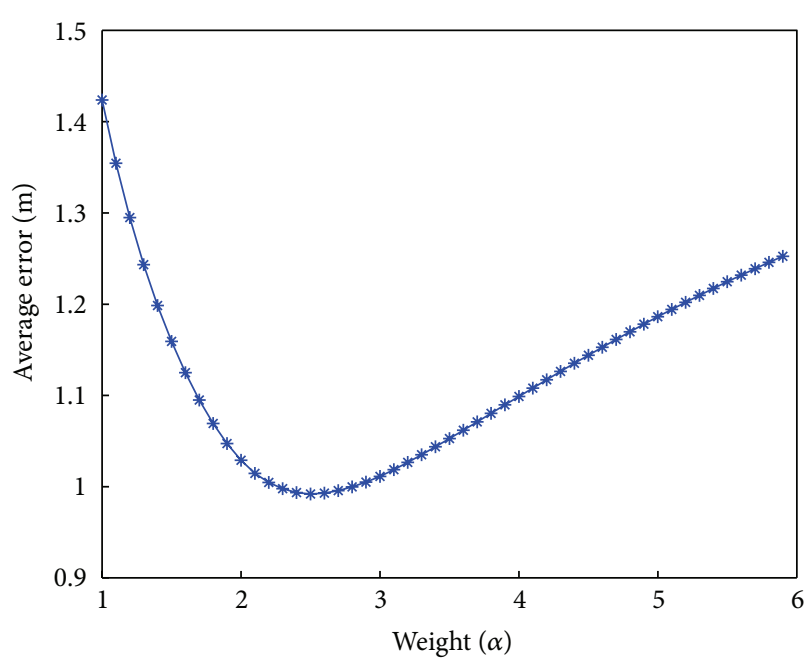

FIGURE 11: The dependence of weighting value on the average location estimation error for path 2 .
TABLE 1: The estimation errors with corresponding variables for Path 1 movement.

\begin{tabular}{|c|c|c|}
\hline Sensing radius & Power-level $(k)$ & Estimation error \\
\hline 7.1 & 1.4 & 0.7001 \\
\hline 7.2 & 1.8 & 0.7801 \\
\hline 7.3 & 1.3 & 0.7001 \\
\hline 7.4 & 1.3 & 0.7001 \\
\hline 7.5 & 1.3 & 0.7001 \\
\hline 7.6 & 1.3 & 0.7001 \\
\hline 7.7 & 1.2 & 0.8337 \\
\hline 7.8 & 1.2 & 0.8337 \\
\hline 7.9 & 1.2 & 0.7800 \\
\hline 8.0 & 1.2 & 0.7435 \\
\hline 8.1 & 1.2 & 0.7435 \\
\hline 8.2 & 1.2 & 0.7435 \\
\hline 8.3 & 1.2 & 0.8352 \\
\hline 8.4 & 1.1 & 0.9099 \\
\hline 8.5 & 1.5 & 0.8576 \\
\hline 8.6 & 1.1 & 0.8062 \\
\hline 8.7 & 2.0 & 0.9888 \\
\hline 8.8 & 1.9 & 0.9548 \\
\hline 8.9 & 1.9 & 0.9334 \\
\hline 9.0 & 1.9 & 0.8808 \\
\hline 9.1 & 1.9 & 0.8836 \\
\hline 9.2 & 1.8 & 0.8497 \\
\hline 9.3 & 1.8 & 0.8497 \\
\hline 9.4 & 1.8 & 0.8283 \\
\hline 9.5 & 1.5 & 0.6297 \\
\hline 9.6 & 1.5 & 0.6297 \\
\hline 9.7 & 1.5 & 0.6140 \\
\hline 9.8 & 1.7 & 0.6139 \\
\hline 9.9 & 1.7 & 0.5925 \\
\hline 10 & 1.7 & 0.5925 \\
\hline
\end{tabular}

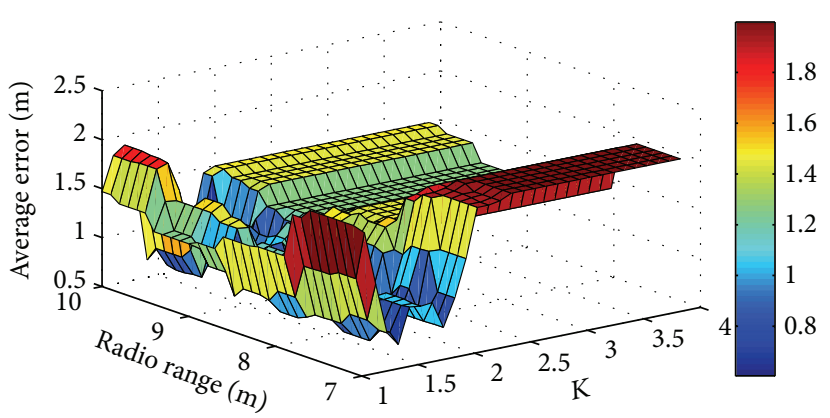

FIGURE 12: Average estimation error with different sensing radii and power-level ratios $k$ for path 1 .

Similar results with different sensing radii and powerlevel increasing ratios $k$ for path 2 case is shown in Figure 13, and the detailed estimation errors with corresponding variables for path 2 case are listed in Table 2. Again, we may take the optimal sensing radius as $9.6 \mathrm{~m}$ and $k$ value as 1.5 for path 2 case from this result. 
TABLE 2: The estimation errors with corresponding variables for Path 2 movement.

\begin{tabular}{lcc}
\hline Sensing radius & Power-level $(k)$ & Estimation error \\
\hline 7.1 & 1.3 & 1.0816 \\
7.2 & 1.3 & 1.0725 \\
7.3 & 1.3 & 1.0607 \\
7.4 & 1.3 & 1.0618 \\
7.5 & 1.3 & 1.1070 \\
7.6 & 1.3 & 1.1575 \\
7.7 & 1.2 & 1.1804 \\
7.8 & 1.2 & 1.1950 \\
7.9 & 1.2 & 1.2324 \\
8.0 & 1.6 & 1.2183 \\
8.1 & 1.6 & 1.1980 \\
8.2 & 1.6 & 1.2118 \\
8.3 & 1.6 & 1.2684 \\
8.4 & 1.6 & 1.2453 \\
8.5 & 1.7 & 1.2698 \\
8.6 & 1.5 & 1.2534 \\
8.7 & 1.6 & 1.2341 \\
8.8 & 1.6 & 1.1755 \\
8.9 & 1.6 & 1.1321 \\
9.0 & 1.6 & 1.1219 \\
9.1 & 1.6 & 1.1225 \\
9.2 & 1.6 & 1.1347 \\
9.3 & 1.5 & 1.0880 \\
9.4 & 1.5 & 1.0358 \\
9.5 & 1.5 & 1.0351 \\
9.6 & 1.5 & 1.0290 \\
9.7 & 1.5 & 1.0532 \\
9.8 & 1.1100 \\
9.9 & 1.1194 \\
10 & 1.1180 \\
\hline
\end{tabular}

4.5. Result of Shadowing Model. In this subsection, the shadowing model is considered, and the simulation is conducted for 100 times to analyze the result. Figure 14 shows the location estimation error with shadowing model for path 1 movement. The sensing radius is set to $9.7 \mathrm{~m}$. From the result in Figure 14, the estimation error is $1 \mathrm{~m}$ with $4.47 \mathrm{~m}$ maximum error and standard deviation 0.72 . On the other hand, the estimation error with shadowing model for path 2 case with $9.6 \mathrm{~m}$ sensing radius is shown in Figure 15. From the result in Figure 15, the estimation error is $1.36 \mathrm{~m}$ with $5.43 \mathrm{~m}$ maximum error and standard deviation 0.77 . The results for path 1 and path 2 for shadowing model are within acceptable range.

4.6. Comparison with Other Schemes. As mentioned earlier, the proposed scheme is based on the low power consumption and computation for moving objecting location tracking. In this sub-section, we therefore, further compare our estimation accuracy with the results of centroid scheme [13]. Figure 16 shows the comparison of location estimation error

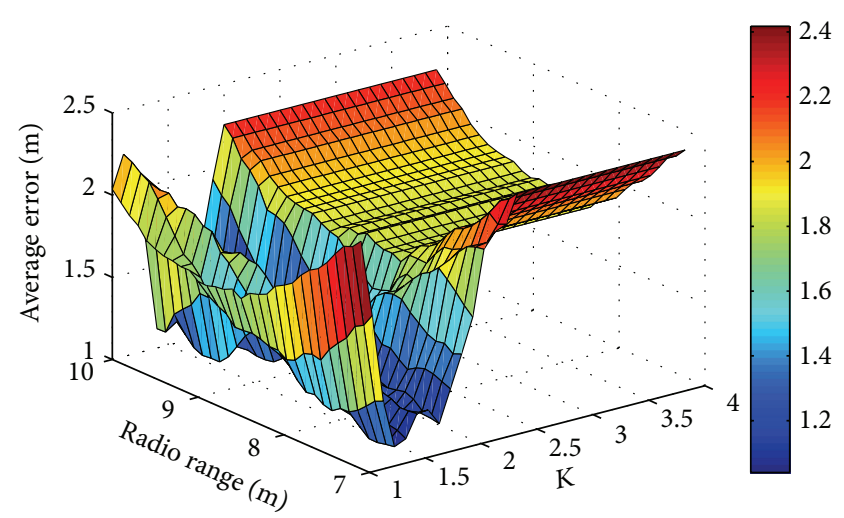

FIGURE 13: Average estimation error with different sensing radii and power-level ratios $k$ for path 2 .

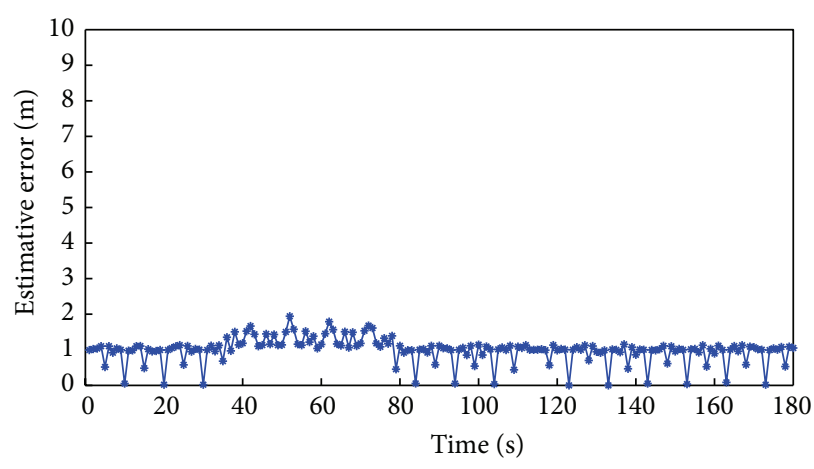

FIGURE 14: The location estimation error with shadowing model for path 1 movement.

between centroid and our proposed schemes for path 1 movement. As we can see from the results of Figure 16, our proposed scheme performs better estimation accuracy than centroid scheme for most radio sensing radii. The proposed scheme comes out with a little bit higher estimation error around sensing radii from $12.5 \mathrm{~m}$ to $13 \mathrm{~m}$. Similarly, the comparison of location estimation error between Centroid and our proposed schemes for path 2 movement is illustrated in Figure 17. Again most results of our proposed scheme show better estimation accuracy than centroid scheme except for the results for radii from $12.6 \mathrm{~m}$ to $13.8 \mathrm{~m}$. However, for $100 \mathrm{~m}$ $\times 100$ m experimental setup, the optimal sensing radius would be around 9.6-9.7 $\mathrm{m}$ from the previous results. Therefore, from practical deployment viewpoint, our proposed scheme does provide much better accuracy as compared with centroid scheme. In general, the estimation error of our proposed mechanism is less than half of that of centroid scheme.

\section{Conclusion}

In this paper, we have developed and proposed the mechanism and procedure to model the location estimation for object tracking in large-scale WSNs. The designed modeling is a simple scheme without complex processing which uses range-free-positioning technology as well as centralized data 


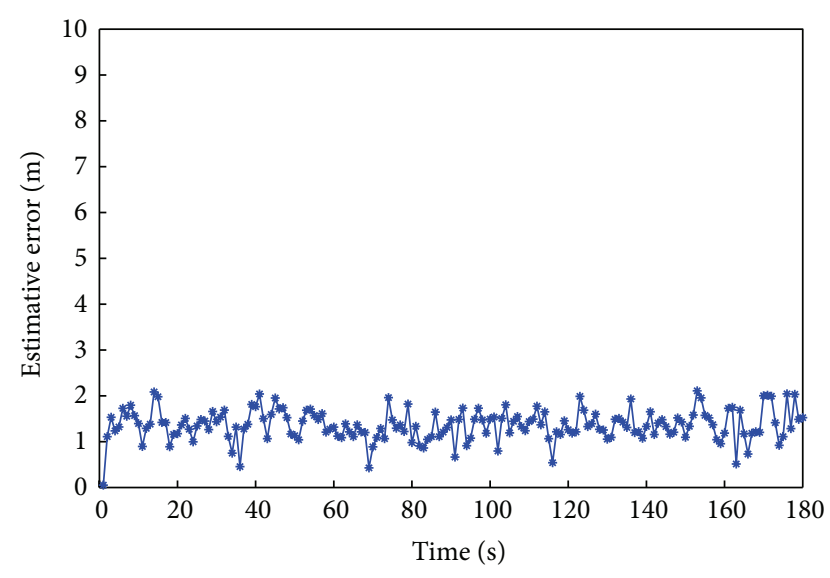

FIGURE 15: The location estimation error with shadowing model for path 2 movement.

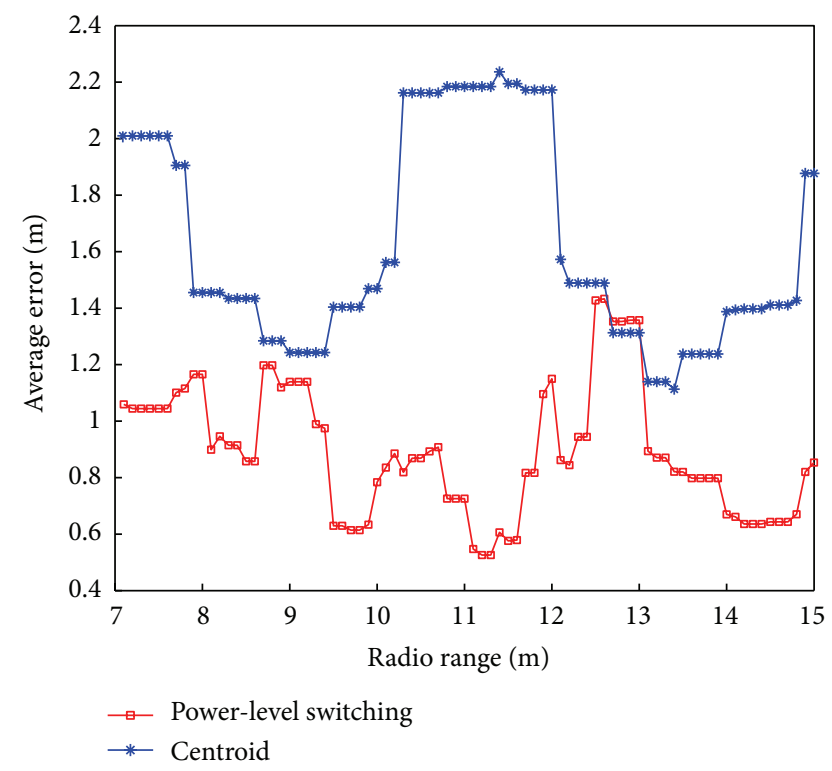

FIGURE 16: The comparison of location estimation error with centroid model for path 1 movement.

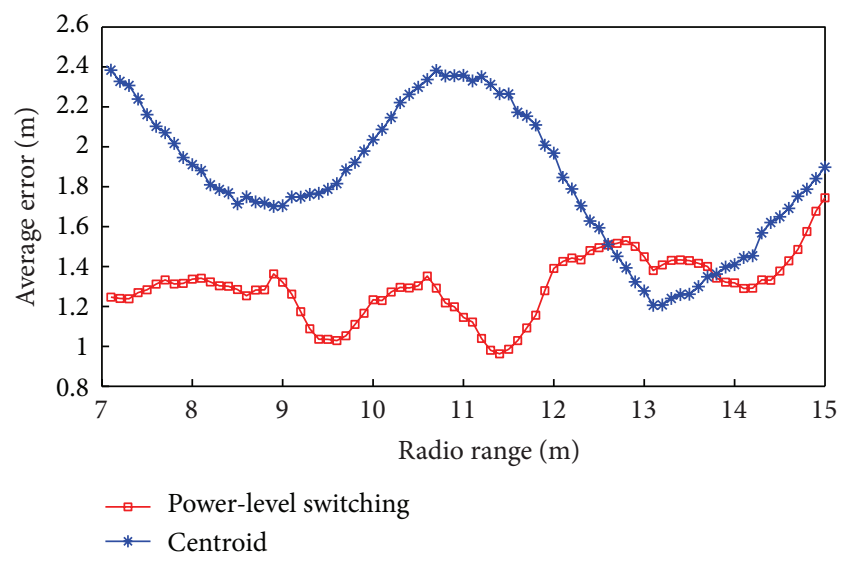

FIGURE 17: The comparison of location estimation error with centroid model for path 2 movement. processing technology with data aggregation idea to reduce the data processing and traffic loads. The proposed positioning model and mechanism are suitable for practical use in large-scale WSNs which are constrained in terms of energy consumption, computation power, and device cost.

We have conducted the simulation and numerical analyses on different variables, such as object moving model, sensing radius, model weighting value $\alpha$, and power-level increasing ratio $k$ of neighboring sensor nodes. The shadowing model was also introduced and analyzed to map the designed scheme to the practical situation. The experimental results showed that the average estimation errors are $0.61 \mathrm{~m}$ and $1.03 \mathrm{~m}$ with optimized sensing radius around $9.7 \mathrm{~m}$ and $9.6 \mathrm{~m}$ for path 1 line movement and path 2 random movement, respectively. We have further compared our proposed model and mechanism with centroid scheme. From practical deployment viewpoint, our proposed scheme does provide much better accuracy as compared with centroid scheme. In general, the estimation error of our proposed mechanism is less than half of that of centroid scheme.

In the future, we will investigate the design of mobile sensing nodes to further reduce the number of deployed nodes. We will also conduct experiments on irregular deployment of sensing nodes to simulate some special scenarios or environments.

\section{Acknowledgments}

This research was partially supported by the National Science Council of Republic of China, Taiwan, under contracts NSC 102-2221-E-324-023, NSC 101-2221-E-142-003, and NSC 992632-E-324-001-MY3.

\section{References}

[1] P. Gao, W. Shi, W. Zhou, H. Li, and X. Wang, "A location predicting method for indoor mobile target localization in wireless sensor networks," International Journal of Distributed Sensor Networks, vol. 2013, Article ID 949285, 11 pages, 2013.

[2] O. G. Adewumi, K. Djouani, and A. M. Kurien, "RSSI based indoor and outdoor distance estimation for localization in WSN," in Proceedings of the IEEE International Conference on Industrial Technology (ICIT '13), pp. 1534-1539, February 2013.

[3] T.-S. Chen, W.-H. Liao, M.-D. Huang, and H.-W. Tsai, "Dynamic object tracking in wireless sensor networks," in Proceedings of the 7th IEEE Malaysia International Conference on Communications, vol. 1, pp. 475-480, November 2005.

[4] C.-Y. Lin and Y.-C. Tseng, "Structures for in-network moving object tracking in wireless sensor networks," in Proceedings of the 1st International Conference on Broadband Networks (BroadNets '04), pp. 718-727, October 2004.

[5] J. Tan and X. Shan, "Spatiotemporal sensor network and mobile robot coordination in constrained environments," in Proceedings of the 6th World Congress on Intelligent Control and Automation (WCICA '06), vol. 1, pp. 391-396, June 2006.

[6] C.-Y. Lin, W.-C. Peng, and Y.-C. Tseng, "Efficient in-network moving object tracking in wireless sensor networks," IEEE Transactions on Mobile Computing, vol. 5, no. 8, pp. 1044-1056, 2006. 
[7] R. R. Brooks, P. Ramanathan, and A. M. Sayeed, "Distributed target classification and tracking in sensor networks," Proceedings of the IEEE, vol. 91, no. 8, pp. 1163-1171, 2003.

[8] B. Hofmann-Wellenhof, H. Lichtenegger, and J. Collins, Global Positioning System: Theory and Practice, Springer, New York, NY, USA, 4th edition, 1997.

[9] M. Vossiek, L. Wiebking, P. Gulden, J. Wieghardt, C. Hoffmann, and P. Heide, "Wireless local positioning," IEEE Microwave Magazine, vol. 4, no. 4, pp. 77-86, 2004.

[10] D. Niculescu and B. Nath, "Ad hoc positioning system (APS) using AOA," in Proceedings of the 22nd Annual Joint Conference on the IEEE Computer and Communications Societies, vol. 3, pp. 1734-1743, April 2003.

[11] A. Awad, T. Frunzke, and F. Dressler, "Adaptive distance estimation and localization in WSN using RSSI measures," in Proceedings of the 10th Euromicro Conference on Digital System Design Architectures, Methods and Tools (DSD '07), pp. 471-478, August 2007.

[12] T. He, C. Huang, B. Lum, J. Stankovic, and T. Adelzaher, "Rangefree localization schemes for large scale sensor networks," in Proceedings of the ACM Annual International Conference on Mobile Computing and Networking (MobiCom '03), September 2003.

[13] N. Bulusu, J. Heidemann, and D. Estrin, "GPS-less low-cost outdoor localization for very small devices," IEEE Personal Communications, vol. 7, no. 5, pp. 28-34, 2000.

[14] Y.-C. Tseng, S.-P. Kuo, H.-W. Lee, and C.-F. Huang, "Location tracking in a wireless sensor network by mobile agents and its data fusion strategies," Computer Journal, vol. 47, no. 4, pp. 448460, 2004.

[15] J. Jeong, T. Hwang, T. He, and D. Du, "MCTA: target tracking algorithm based on minimal contour in wireless sensor networks," in Proceedings of the 26th IEEE International Conference on Computer Communications (INFOCOM '07), pp. 2371-2375, May 2007.

[16] S. Capkun, M. Hamdi, and J. Hubaux, "GPS-free positioning in mobile ad-hoc networks," in Proceedings of the 34th Annual Hawaii International Conference on System Sciences, pp. 34813490, January 2001.

[17] K.-F. Ssu, C.-H. Ou, and H. C. Jiau, "Localization with mobile anchor points in wireless sensor networks," IEEE Transactions on Vehicular Technology, vol. 54, no. 3, pp. 1187-1197, 2005.

[18] B. Krishnamachari, D. Estrin, and S. Wicker, "The impact of data aggregation in wireless sensor networks," in Proceedings of the 22nd International Conference on Distributed Computing Systems Workshops, pp. 575-578, July 2002.

[19] H.-C. Chu and R.-H. Jan, "A GPS-less, outdoor, self-positioning method for wireless sensor networks," Ad Hoc Networks, vol. 5, no. 5, pp. 547-557, 2007.

[20] H. C. Chu, L. H. Chang, H. W. Yu, J. J. Liaw, and Y. H. Lai, “Target tracking in wireless sensor networks with guard nodes," Journal of Internet Technology, vol. 11, no. 7, 2010.

[21] W. Kim, J. Park, J. Yoo, H. J. Kim, and C. G. Park, “Target localization using ensemble support vector regression in wireless sensor networks," IEEE Transactions on Cybernetics, vol. 43, no. 4, pp. 1189-1198, 2013.

[22] T. Camp, J. Boleng, and V. Davies, "A survey of mobility models for ad hoc network research," Wireless Communications and Mobile Computing, vol. 2, no. 5, pp. 483-502, 2002. 


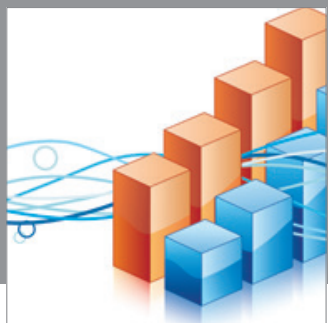

Advances in

Operations Research

mansans

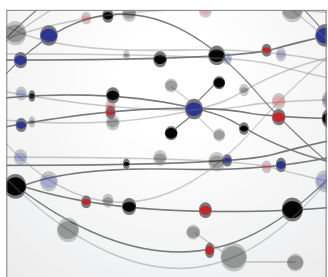

The Scientific World Journal
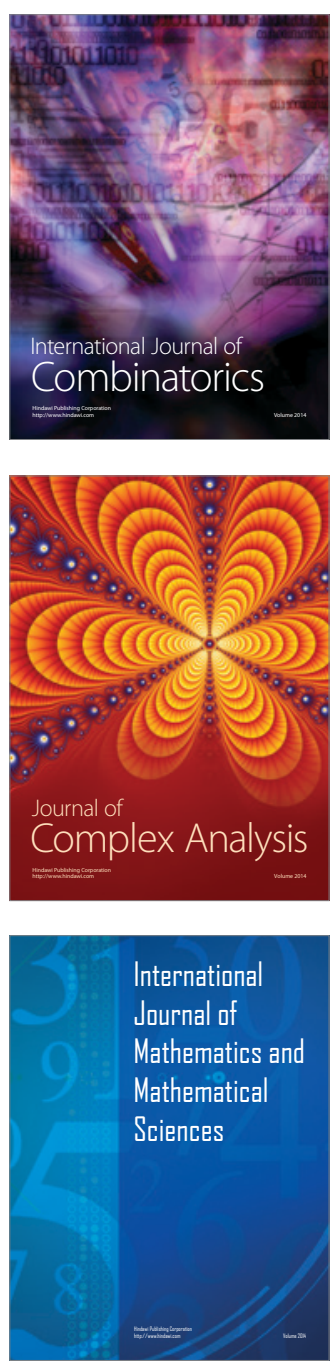
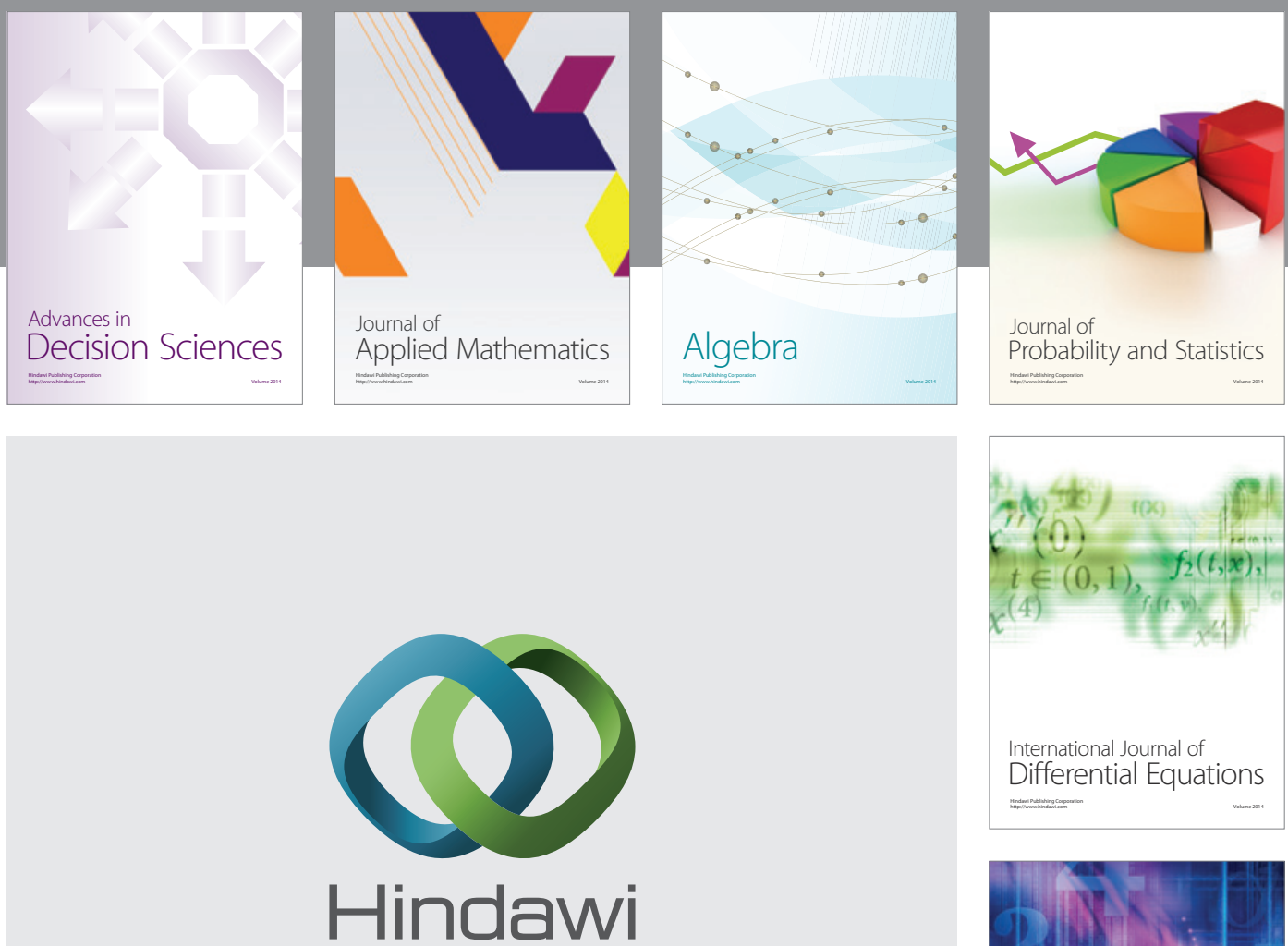

Submit your manuscripts at http://www.hindawi.com
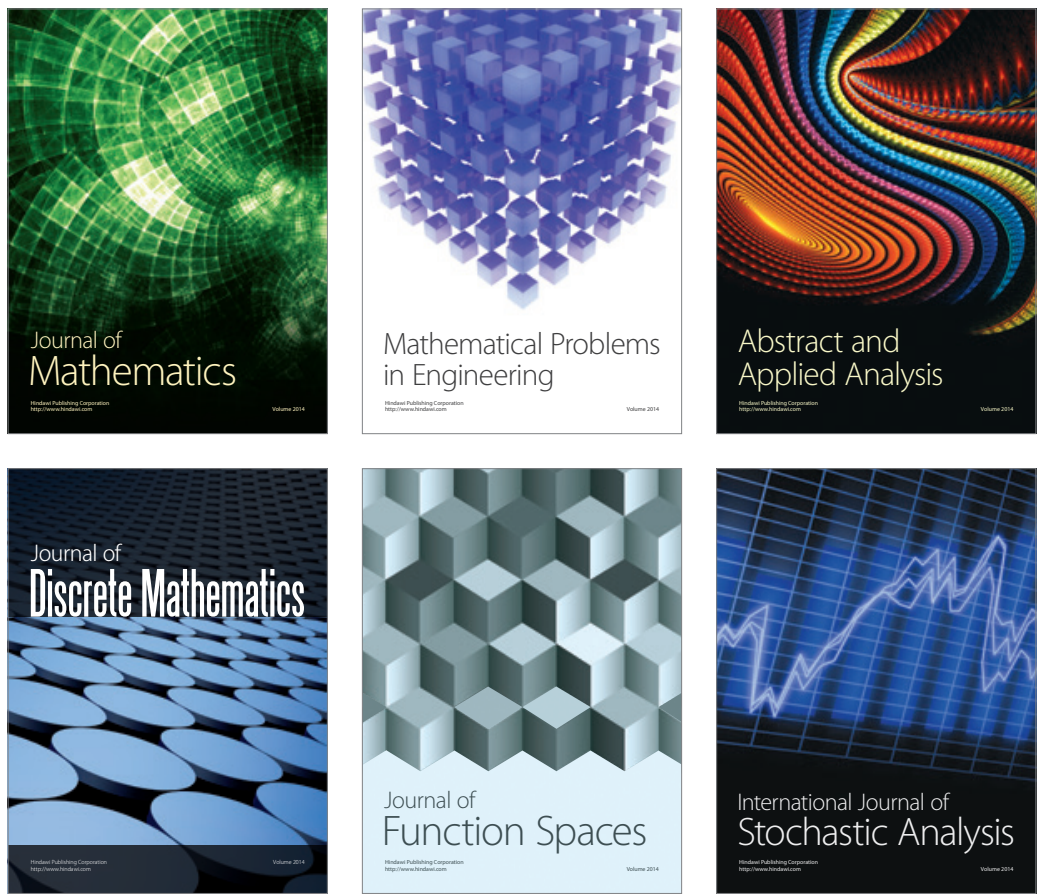

Journal of

Function Spaces

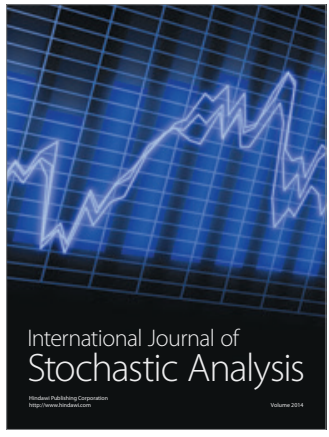

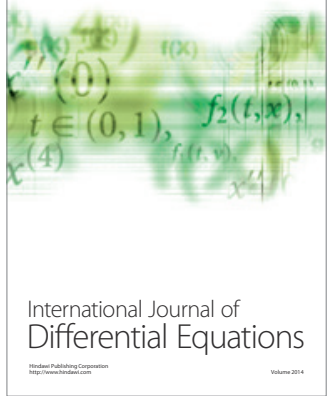
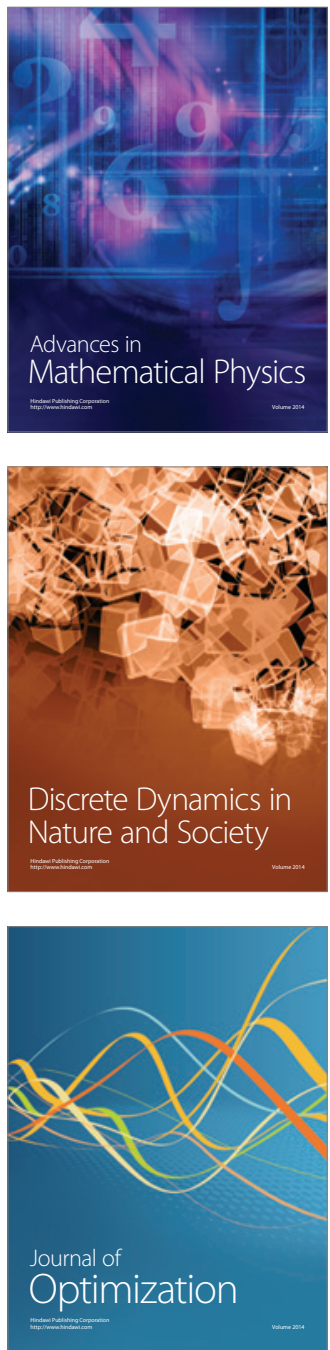